\title{
A constant in pluripotential theory
}

by ZBigniew BŁocki (Kraków)

Abstract. We compute the constant

$$
\sup \left\{\frac{1}{\operatorname{deg} P}\left(\max _{S} \log |P|-\int_{S} \log |P| d \sigma\right): P \text { a polynomial in } \mathbb{C}^{n}\right\},
$$

where $S$ denotes the euclidean unit sphere in $\mathbb{C}^{n}$ and $\sigma$ its unitary surface measure.

Let $S$ be the euclidean unit sphere in $\mathbb{C}^{n}$ and $\sigma$ its unitary surface measure. Setting

$$
L:=\left\{u \in \operatorname{PSH}\left(\mathbb{C}^{n}\right): \sup \left\{u(z)-\log _{+}|z|: z \in \mathbb{C}^{n}\right\}<\infty\right\}
$$

we define

$$
c_{n}:=\sup \left\{\max _{S} u-\int_{S} u d \sigma: u \in L\right\} .
$$

The aim of this note is to find the exact value of the constant $c_{n}$ (for its applications see [2]). We start with the following

Lemma 1. For $a \in \mathbb{C}$ we have

$$
\int_{S} \log \left|z_{n}+a\right| d \sigma(z)= \begin{cases}-\frac{1}{2} \sum_{j=1}^{n-1} \frac{1}{j}\left(1-|a|^{2}\right)^{j} & \text { if }|a| \leq 1, \\ \log |a| & \text { if }|a| \geq 1 .\end{cases}
$$

Proof. The function $z \mapsto \log \left|z_{n}+a\right|$ is harmonic in $\{|z|<|a|\}$, thus for $|a| \geq 1$ the statement is obvious. Now let $|a|<1$. Without loss of generality we may assume $a \geq 0$. We have

$$
\int_{S} \log \left|z_{n}+a\right| d \sigma(z)=\frac{n-1}{\pi} \int_{0}^{1} r\left(1-r^{2}\right)^{n-2} \int_{0}^{2 \pi} \log \left|r e^{i t}+a\right| d t d r
$$

1991 Mathematics Subject Classification: Primary 31C10, 32F05. 
(see [3], p. 15). Using the equality

$$
\frac{1}{2 \pi} \int_{0}^{2 \pi} \log \left|r e^{i t}+a\right| d t=\max \{\log r, \log a\}
$$

we see that

$$
\begin{aligned}
\int_{S} \log \left|z_{n}+a\right| d \sigma(z)= & 2(n-1) \log a \int_{0}^{a} r\left(1-r^{2}\right)^{n-2} d r \\
& +2(n-1) \int_{a}^{1} r\left(1-r^{2}\right)^{n-2} \log r d r \\
= & -\log a\left[\left(1-r^{2}\right)^{n-1}\right]_{0}^{a} \\
& +\left[-\left(1-r^{2}\right)^{n-1} \log r+\log r+\frac{1}{2} \sum_{j=1}^{n-1} \frac{1}{j}\left(1-r^{2}\right)^{j}\right]_{a}^{1} \\
= & -\frac{1}{2} \sum_{j=1}^{n-1} \frac{1}{j}\left(1-a^{2}\right)^{j} .
\end{aligned}
$$

Lemma 2. For every $n \geq 2$ there exists a unique $\varrho_{n} \in(0,1)$ such that $\left(1+\varrho_{n}\right)\left(1-\varrho_{n}^{2}\right)^{n-1}=1$. Moreover, $\left\{\varrho_{n}\right\}$ decreases to 0 .

Proof. For $\varrho \in[0,1]$ let

$$
f_{n}(\varrho):=(1+\varrho)\left(1-\varrho^{2}\right)^{n-1}-1 .
$$

We have

$$
f_{n}^{\prime}(\varrho)=-(2 n-1)(1+\varrho)\left(1-\varrho^{2}\right)^{n-2}\left(\varrho-\frac{1}{2 n-1}\right) .
$$

Hence $f_{n}(0)=0, f_{n}$ increases in $(0,1 /(2 n-1))$, decreases in $(1 /(2 n-1), 1)$ and $f_{n}(1)=-1$. Thus the first statement is clear. Now since $f_{n+1}\left(\varrho_{n}\right)<$ $f_{n}\left(\varrho_{n}\right)=0$ we see that the sequence $\left\{\varrho_{n}\right\}$ is decreasing. Let then $\varepsilon:=\lim \varrho_{n}$. Since

$$
0=\lim f_{n}\left(\varrho_{n}\right) \leq \lim 2\left(1-\varepsilon^{2}\right)^{n-1}-1
$$

we conclude that $\varepsilon$ must be equal to 0 .

THEOREM. The supremum in (1) is attained for the function $z \mapsto$ $\log \left|z_{n}+\varrho_{n}\right|$ where $\varrho_{1}=1$ and for $n \geq 2$, $\varrho_{n}$ is given by Lemma 2 . Therefore by Lemma 1 we have

$$
\begin{aligned}
c_{1} & =\log 2, \\
c_{n} & =\log \left(1+\varrho_{n}\right)+\frac{1}{2} \sum_{j=1}^{n-1} \frac{1}{j}\left(1-\varrho_{n}^{2}\right)^{j} \quad \text { for } n \geq 2 .
\end{aligned}
$$


Proof. Let $u \in L$. First we want to show that it is enough to take functions in the supremum which depend on one variable. For $n \geq 2$ we have

$$
\int_{S} u d \sigma=\int_{D} \int_{\Gamma_{z_{n}}} u\left(z^{\prime}, z_{n}\right) d \sigma_{z_{n}}\left(z^{\prime}\right) A\left(\left|z_{n}\right|\right) d \lambda\left(z_{n}\right),
$$

where $D$ is the unit disc in $\mathbb{C}, \lambda$ its Lebesgue measure, $z^{\prime}=\left(z_{1}, \ldots, z_{n-1}\right) \in$ $\mathbb{C}^{n-1}$,

$$
\Gamma_{z_{n}}:=\left\{\left(z^{\prime}, z_{n}\right) \in \mathbb{C}^{n}:\left|z^{\prime}\right|^{2}=1-\left|z_{n}\right|^{2}\right\} \subset S,
$$

$\sigma_{z_{n}}$ the unitary surface measure on $\Gamma_{z_{n}}$ and

$$
A(r):=\frac{n-1}{\pi}\left(1-r^{2}\right)^{n-2} .
$$

By subharmonicity of the function $z^{\prime} \mapsto u\left(z^{\prime}, z_{n}\right)$ we have

$$
\int_{\Gamma_{z_{n}}} u\left(z^{\prime}, z_{n}\right) d \sigma_{z_{n}}\left(z^{\prime}\right) \geq u\left(0, z_{n}\right)
$$

and therefore

$$
\int_{S} u d \sigma \geq \int_{S} u\left(0, z_{n}\right) d \sigma(z)
$$

Since $\sigma$ is invariant under unitary transformations we may assume that $\max _{S} u=u(0, \ldots, 0,1)$, hence

$$
\max _{S} u-\int_{S} u d \sigma \leq \max _{\left|z_{n}\right|=1} u\left(0, z_{n}\right)-\int_{S} u\left(0, z_{n}\right) d \sigma(z) .
$$

We may thus consider only functions from $L$ depending on the one variable $z_{n}$. Define

$$
\begin{aligned}
c_{n}^{\prime} & :=\sup \left\{\frac{1}{d}\left(\max _{S} \log |P|-\int_{S} \log |P| d \sigma\right): P \in \mathcal{P}_{d}\left(\mathbb{C}^{n}\right)\right\} \\
& =\sup \left\{\frac{1}{d}\left(\max _{\left|z_{n}\right|=1} \log \left|P\left(z_{n}\right)\right|-\int_{S} \log \left|P\left(z_{n}\right)\right| d \sigma(z)\right): P \in \mathcal{P}_{d}(\mathbb{C})\right\}
\end{aligned}
$$

where $\mathcal{P}_{d}\left(\mathbb{C}^{n}\right)$ denotes the set of all polynomials in $\mathbb{C}^{n}$ of degree $\leq d$. If $P(\zeta)=\alpha\left(\zeta+a_{1}\right) \ldots\left(\zeta+a_{d}\right)$ then

$$
\begin{aligned}
& \frac{1}{d}\left(\max _{\left|z_{n}\right|=1} \log \left|P\left(z_{n}\right)\right|-\int_{S} \log \left|P\left(z_{n}\right)\right| d \sigma(z)\right) \\
& \leq \frac{1}{d} \sum_{k=1}^{d}\left(\max _{\left|z_{n}\right|=1} \log \left|z_{n}+a_{k}\right|-\int_{S} \log \left|z_{n}+a_{k}\right| d \sigma(z)\right) \\
& \leq \max _{k=1, \ldots, d}\left(\max _{\left|z_{n}\right|=1} \log \left|z_{n}+a_{k}\right|-\int_{S} \log \left|z_{n}+a_{k}\right| d \sigma(z)\right) .
\end{aligned}
$$


Therefore

$$
c_{n}^{\prime}=\sup \left\{\max _{\left|z_{n}\right|=1} \log \left|z_{n}+\varrho\right|-\int_{S} \log \left|z_{n}+\varrho\right| d \sigma(z): \varrho \in[0,1]\right\}
$$

(since for $\varrho>1$ one has $\log (1+\varrho)-\log \varrho<\log 2$, and by Lemma 1 , it is enough to take only $\varrho \in[0,1]$ in the supremum). For $\varrho \in[0,1]$ put

$$
g_{n}(\varrho):=\max _{\left|z_{n}\right|=1} \log \left|z_{n}+\varrho\right|-\int_{S} \log \left|z_{n}+\varrho\right| d \sigma(z) .
$$

By Lemma 1

$$
g_{n}(\varrho)=\log (1+\varrho)+\frac{1}{2} \sum_{j=1}^{n-1} \frac{1}{j}\left(1-\varrho^{2}\right)^{j} .
$$

Therefore $g_{1}^{\prime}(\varrho)=1 /(1+\varrho)$ and for $n \geq 2$

$$
\begin{aligned}
& g_{n}^{\prime}(0)=1, \\
& g_{n}^{\prime}(\varrho)=\frac{(1+\varrho)\left(1-\varrho^{2}\right)^{n-1}-1}{(1+\varrho) \varrho} \quad \text { for } \varrho>0, \\
& g_{n}^{\prime}(1)=-1 / 2 .
\end{aligned}
$$

Hence, by Lemma 2 it is enough to prove that $c_{n}^{\prime}=c_{n}$. Of course $c_{n}^{\prime} \leq c_{n}$, let us then take any $u \in L$. By the approximation property of $L$ (see [4]) there exists a sequence of polynomials $\left\{P_{k}\right\}$ such that $P_{k} \in \mathcal{P}_{k}$ and

$$
u=\left(\limsup _{k \rightarrow \infty} \frac{1}{k} \log \left|P_{k}\right|\right)^{*}
$$

$\left(v^{*}\right.$ denotes the upper regularization of $\left.v\right)$. Now Fatou's Lemma gives

$$
\max _{S} u-\int_{S} u d \sigma \leq \limsup _{k \rightarrow \infty} \frac{1}{k}\left(\max _{S} \log \left|P_{k}\right|-\int_{S} \log \left|P_{k}\right| d \sigma\right) .
$$

The proof is complete.

Put

$$
H:=\left\{v \in \operatorname{PSH}\left(\mathbb{C}^{n}\right): v(a z)=v(z)+\log |a| \text { for all } a \in \mathbb{C}, z \in \mathbb{C}^{n}\right\}
$$

and define

$$
\kappa_{n}:=\sup \left\{\max _{S} v-\int_{S} v d \sigma: v \in H\right\} .
$$

Then $H \subset L$ and

$$
\kappa_{n}=-\int_{S} \log \left|z_{n}\right| d \sigma(z)=\frac{1}{2} \sum_{j=1}^{n-1} \frac{1}{j}
$$

(see [4] and [1]). 
Corollary. $c_{1}=\log 2, \kappa_{n}<c_{n}<\kappa_{n}+\log \left(1+\varrho_{n}\right)$ for $n \geq 2$, $\lim _{n \rightarrow \infty}\left(c_{n}-\kappa_{n}\right)=0$ and $\left\{c_{n}\right\}$ is increasing.

Proof. Everything but the last statement is clear. Taking $g_{n}$ defined by (2) we see that $g_{n} \leq g_{n+1}$, hence

$$
c_{n}=\max _{[0,1]} g_{n}<\max _{[0,1]} g_{n+1}=c_{n+1}
$$

\section{References}

[1] H. Alexander, Projective capacity, in: Ann. of Math. Stud. 100, Princeton Univ. Press, 1981, 3-27.

[2] J.-P. Demailly, Potential theory in several complex variables, preprint, 1989.

[3] W. Rudin, Function Theory in the Unit Ball of $\mathbb{C}^{n}$, Springer, 1980.

[4] J. Siciak, Extremal plurisubharmonic functions and capacities in $\mathbb{C}^{n}$, Sophia Kokyuroku in Math. 14 (1982).

INSTITUTE OF MATHEMATICS

JAGIELLONIAN UNIVERSITY

REYMONTA 4

30-059 KRAKÓW, POLAND

E-MAIL: UMBLOCKI@PLKRCY11.BITNET

Reçu par la Rédaction le 18.3.1991 